\title{
Dry-spinning of cellulose nanocrystal/polylactic acid composite fibers
}

1 Caitlyn M. Clarkson MSc

Graduate student, School of Materials Engineering, Purdue University, West Lafayette, IN, USA (Orcid:0000-0001-9689-0842)
2 Jeffrey P. Youngblood PhD

Associate Professor, School of Materials Engineering, Purdue University, West Lafayette, IN, USA (corresponding author: jpyoungb@purdue.edu) (Orcid:0000-0002-8720-8642)
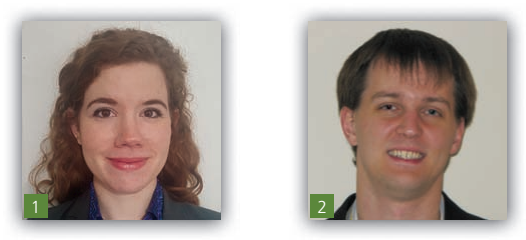

In the present study, the authors explored the viability of dry-spinning to produce cellulose nanocrystal (CNC) and polylactic acid composite fibers from dimethylformamide solutions. Fiber surface morphology, crystallinity and mechanical properties were assessed for fibers with 0, 1, 3 and $5 \mathrm{wt} \%$ CNCs. The fiber morphology transitioned from smooth to sharkskin to severe melt fracture with increasing extrusion speed. Surface morphology appeared to be weakly dependent on CNC content and addition of CNCs did not reduce melt fracture. Addition of $1 \mathrm{wt} \%$ CNC resulted in the highest crystallinity, an approximately $30 \%$ increase for the dry-spun composite fibers. Fibers exhibited a mixture of ductile and brittle-like behavior during mechanical testing. The elastic modulus and strength at failure were measured for three fiber diameter ranges and for increasing CNC content. A maximum average elastic modulus of $6.5 \mathrm{GPa}$ and $100 \mathrm{MPa}$ increase in strength at failure are reported for dry-spun composite fibers.

\section{Notation}

E elastic modulus

$L / D \quad$ length/diameter ratio

$T_{\text {cc }} \quad$ cold crystallization temperature

$T_{\mathrm{m}} \quad$ melting point

$V_{\mathrm{f}} \quad$ volume fraction

$x_{\mathrm{cnc}} \quad$ weight fraction of cellulose nanocrystals (CNCs) in the polylactic acid/CNC fiber

$\Delta H_{\mathrm{c}} \quad$ enthalpy of cold crystallization

$\Delta H_{\mathrm{m}} \quad$ enthalpy of melting

$\Delta H_{\mathrm{m}}^{\circ} \quad$ theoretical enthalpy of melting

$\eta \quad$ Halpin-Tsai parameter

$\chi \quad$ degree of crystallinity

\section{Introduction}

Nanocellulose is an organic nanoparticle with dimensions of $5-20 \mathrm{~nm}$ width and $20 \mathrm{~nm}-10 \mu \mathrm{m}$ length that is derived from various, abundant biomass sources. ${ }^{1-3}$ Nanocellulose refers to a broad class of biomaterials which includes cellulose nanocrystals (CNCs), cellulose nanofibrils, microfibrillated cellulose and bacterial cellulose. ${ }^{1} \mathrm{CNCs}$ have generated much interest because these nanoparticles are rigid whiskers, have high crystallinity (54-88\%) and exhibit various desirable properties. ${ }^{1,3}$ CNCs have been shown to have high modulus, strength and thermal conductivity and low coefficients of thermal and hygroscopic expansions. ${ }^{1,3-6}$
Fiber spinning is commercially used to produce many synthetic fibers. Three primary commercial techniques are described in the literature, which are melt-spinning and the two primary solution processes: dry-spinning and wet-spinning. Melt-spinning produces fibers at the highest production rate and is the simplest process. The polymer is melted and extruded through a spinneret into a cooler, where it then undergoes any number of postprocessing steps. Similarly, in dry-spinning, solutions of polymer and solvent are extruded from a spinneret. However, dry-spinning is a more complex process as solidification depends on solvent evaporation, which in turn depends on many variables, including the solution composition and concentration of constituents and processing variables such as temperature. The slowest process is wet-spinning, where the solution is extruded into a miscible solvent bath and solidification occurs by diffusion of the fiber solvent into the bath solvent. These processes offer different advantages. For instance, solution spinning processes offer lower operation temperatures and the ability to process polymers that do not melt or flow easily.

Nanocellulose-reinforced polymer composites have been explored for fiber applications due to the large stiffness and strength gains in low-cost, commodity polymers. ${ }^{6-9}$ Solution spinning processes are the primary method for producing $\mathrm{CNC}$ nanocomposite fibers. These studies focused primarily on polymers soluble in water and watermiscible organic solvents such as acetone/dimethylacetamide and dimethylformamide (DMF) to address challenges. ${ }^{6-13}$ A study on 
polyvinyl alcohol and $\mathrm{CNC}$ composite fibers produced a maximum $20 \%$ increase in the tensile strength at only $5 \mathrm{wt} \% \mathrm{CNC}$ due to the orientation of the nanocellulose filler. ${ }^{14}$ In cellulose acetate (CA) fibers, the elastic modulus increased by $637 \%$ and the tensile strength increased by $137 \%$ with the addition of $34 \mathrm{wt} \% \mathrm{CNC}^{7}$ However, the maximum alignment was achieved around $30 \mathrm{wt} \%$, after which the alignment was constant and $\mathrm{CNC}$ content alone determined the mechanical performance. ${ }^{7}$ The $\mathrm{CNC}$ concentration at which the transition from alignment-dependent to CNC-loading-controlled properties occurs varies between polymers as aggregation of the nanocellulose will change with polymer system and processing. ${ }^{6,7,12}$ A ductile-to-brittle failure transition may accompany the aforementioned change as was observed in dry-spun $\mathrm{CA} / \mathrm{CNC}$ fibers with the addition of $\mathrm{CNC}$, and a reduction in strain at failure was observed at as little as $2 \cdot 5 \mathrm{wt} \% \mathrm{CNC}^{8}$

Polylactic acid (PLA) is a sustainably sourced alternative to many industrially available polymers, but it is insufficient in mechanical performance. PLA fibers are primarily melt-spun, but dryspinning of PLA has been explored previously from solutions of chloroform, chloroform/toluene and tetrahydrofuran. ${ }^{15-20}$ Tensile strengths as high as $2.3 \mathrm{GPa}$ and elastic moduli as high as $15 \mathrm{GPa}$ have been observed for dry-spun PLA fibers. ${ }^{18,19,21}$ However, PLA fibers typically have poor mechanical properties and are weak, have low stiffness and are brittle. Additionally, melt fracture and heavy surface texturing were observed in dry-spun fibers. ${ }^{16,18,19,21}$ Alternatively, direct injection of a $\mathrm{CNC} /$ water solution into a polymer melt has been employed to produce fibers with the end goal of creating films. ${ }^{22}$ This study reported good dispersion in the fibers; however, the introduction of water into PLA causes degradation of molecular weight as the polymer undergoes hydrolytic cleavage. ${ }^{23}$

In the present study, dry-spinning of CNC/PLA composite fibers (as opposed to melt-spinning and wet-spinning) was explored as an option for producing CNC composite fibers as it could enhance $\mathrm{CNC}$ dispersion in the polymer matrix for unmodified $\mathrm{CNCs}$, provide lower processing temperatures which are essential to prevent thermal degradation of $\mathrm{CNCs}$ and eliminate water from PLA processing, which can degrade molecular weight. To understand the viability of this process for producing high-strength, high-stiffness fibers from CNC/PLA, fibers were produced and the surface morphology, crystallinity and mechanical properties of the reinforced fibers were investigated. To the best of the authors' knowledge, these properties and this system have not yet been reported.

\section{Experimental methods and materials}

\subsection{Materials}

CNC slurry at $11 \cdot 8 \mathrm{wt} \%$ (batch 2014-FPL-CNC-064, $1 \mathrm{wt} \%$ sulfur on dried sodium $\mathrm{CNC}$ form) in water was received from the University of Maine (Orono, Maine, USA), the distributor for nanocellulose manufactured by the US Department of Agriculture Forest Service - Forest Products Laboratory (Madison, Wisconsin,
USA), who produces the material. DMF was purchased from SigmaAldrich (St Louis, Missouri, USA). NatureWorks 6202D fiber-grade PLA for fibers and non-wovens was purchased from a distributor, Jamplast Management Co. (Ellisville, Missouri, USA). Resin pellets were dried at $80^{\circ} \mathrm{C}$ for $24 \mathrm{~h}$ prior to use. DMF was dried over molecular sieves for 2-3d prior to use. Solvent exchange of the $\mathrm{CNCs}$ from water into DMF was performed on a rotary evaporator at 50 Torr to get a solution of approximately $8.5 \mathrm{wt} \% \mathrm{CNC}$. Thermogravimetric analysis was used to confirm that water had been removed from the solvent/CNC solution because water can initiate chain scission in PLA and thus lower the molecular weight.

CNC/PLA solutions were prepared at a fixed concentration of polymer in solution, $30 \mathrm{wt} \%$, and varying $\mathrm{CNC}$ content to achieve final concentrations of 1,3 and $5 \mathrm{wt} \% \mathrm{CNC}$ in the composite fiber. Solutions were prepared by first mixing the appropriate quantity of $\mathrm{CNC} / \mathrm{DMF}$ solution into the quantity of DMF needed to make a $30 \mathrm{wt} \%$ PLA solution. A homogenous solution was achieved by first mechanically mixing the CNC/DMF solutions and then ultrasonicating to disperse the $\mathrm{CNCs}$ further in solution. Ultrasonication on a Branson sonifier was performed for $1 \mathrm{~min}$ at $30 \%$ amplitude with a $1 \mathrm{~s}$ pulse and $1 \mathrm{~s}$ rest to avoid damaging the CNCs. Under vigorous mechanical stirring, the final concentration of PLA in DMF was achieved by elevating the temperature to $80-90^{\circ} \mathrm{C}$ while dissolving the polymer. Higher concentrations of $\mathrm{CNC}$ could not be achieved because of a large increase in solution viscosity that inhibited mechanical mixing of the polymer pellets into solution.

\subsection{Fiber spinning}

Direct-winding fiber spinning was used to produce dry-spun fibers. Fiber spinning was performed on an Xplore $5 \mathrm{cc}$ twin-screw microcompounder with a continuous-feed hopper, $90^{\circ}$ turn and funnel-tapered (conical V-taper) $250 \mu \mathrm{m}$ orifice. A multipletemperature profile was employed for spinning where the top-tobottom barrel temperatures were 100,110 and $115^{\circ} \mathrm{C}$. The polymer solutions were loaded into the prewarmed barrel and allowed to compound at low speed ( 5 revolutions per minute $(\mathrm{rpm})$ ) for a maximum of $2 \mathrm{~h}$ until a spinnable viscosity was achieved. The extrusion speed of the fibers was varied by altering the screw speed of the compounder; screw speeds of 1, 5, 25, 50 and 100 rpm were initially investigated. Fibers were collected directly from the orifice during the spinning process on a winding mandrel. The take-up speed of the winding mandrel was $150 \mathrm{rpm}$ and was held fixed for all processes. Prior to testing, all fibers were dried in a vacuum oven at $70^{\circ} \mathrm{C}$ for several hours to ensure that all residual solvent and any water absorbed from the atmosphere were removed.

\subsection{Surface morphology and fiber diameter}

Scanning electron microscopy (SEM) was utilized to image fiber surfaces on an XL40 field emission scanning electron microscope. Samples were prepared by first sputter-coating surfaces with gold-palladium to provide a conductive barrier to dissipate charge from the organic media. For high-resolution images of organic fibers, a $3-5 \mathrm{keV}$ accelerating voltage and a working distance of $10-15 \mathrm{~mm}$ were selected. 
Dry-spinning of cellulose nanocrystal/

polylactic acid composite fibers

Clarkson and Youngblood
Optical microscopy images of as-spun fibers were taken on a Carl Zeiss (Axio Observer A1) inverted light microscope. The spinning conditions produced fibers with diameters from 20 to $80 \mu \mathrm{m}$. Individual fiber diameters were measured for tensile test analysis.

\subsection{Crystallinity of as-spun fibers}

Thermal data were collected on a Thermal Analysis (TA) Instruments Q2000 differential scanning calorimeter (DSC). Heat-cool-heat temperature ramps were conducted at $10^{\circ} \mathrm{C} / \mathrm{min}$ in nitrogen. The average sample size was $6 \pm 2 \mathrm{mg}$ and three samples were used per experiment.

\subsection{Tensile properties}

Tensile testing of the fibers was performed on a TA Instruments Q800 dynamic mechanical analyzer. Stress-strain data were collected from a force ramp at $0.3 \mathrm{~N} / \mathrm{min}$ and $0.001 \mathrm{~N}$ preload. To avoid pinching off during clamping and make sample loading easier, the

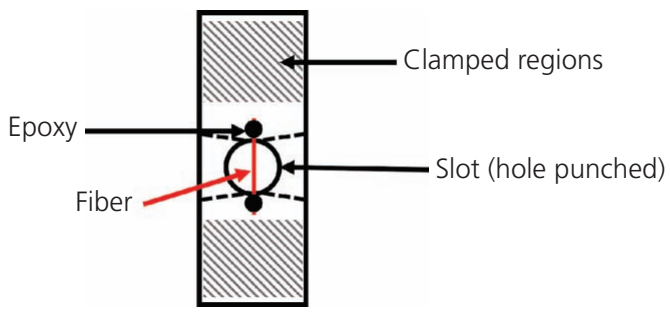

Figure 1. Schematic diagram of carding for testing polymer fibers carding configuration in Figure 1 was adapted to conduct tensile tests. ${ }^{24}$ Fibers were selected at random from dry fiber bundles, and the fiber diameter was measured prior to carding to screen specimens for fiber diameter and group them for easier comparison of experimental parameters. The pattern in Figure 1 was printed onto heavy cardstock to use as a template, and a hole punch was used to remove the center of the cardstock. After screening, the fibers were sectioned into $25.4 \mathrm{~mm}$ pieces for carding. The $25.4 \mathrm{~mm}$ long pieces were secured along the primary axis of the template and highstrength epoxy was used to secure the fiber. The final gauge was $6 \cdot 2 \pm 1.1 \mathrm{~mm}$ length. From optical microscopy images, the true gauge length and minimum diameter of the fiber were recorded for calculation of the engineering stress and engineering strain.

\section{Results and discussion}

\subsection{Surface morphology}

Mild to moderate melt fracture was observed on dry-spun fibers as seen in Figure 2. By tuning the extrusion speed relative to the takeup/winding speed by adjusting the compounder screw speed, the surface morphology of the fiber was seen to change, as seen in Figure 2. For slow screw speeds, the fiber morphology was relatively smooth with random dimpling across the fibers. By increasing the screw speed, the surface textures became much rougher with the onset of sharkskin (Figures 2(b) and 2(e)), which transitioned to more moderate distortion at $100 \mathrm{rpm}$ screw speed (Figure 2(c)). Large-scale distortion, such as gross melt fracture, is thought to occur when an instability in the die forms that cannot be resolved. ${ }^{25}$ For surface textures such as sharkskin, the instability is thought to occur

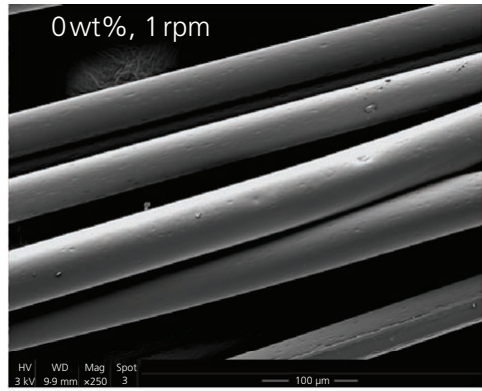

(a)

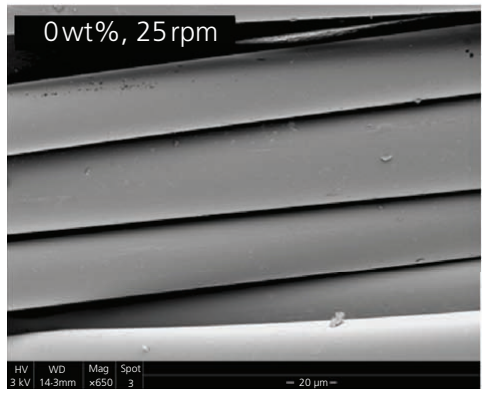

(d)

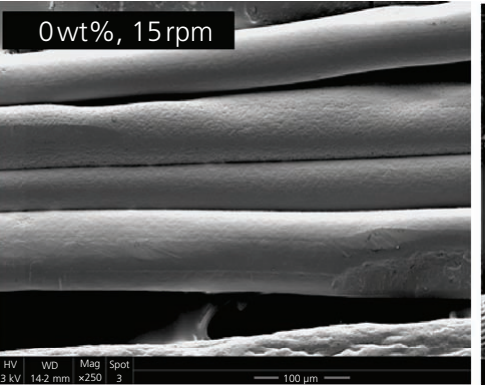

(b)

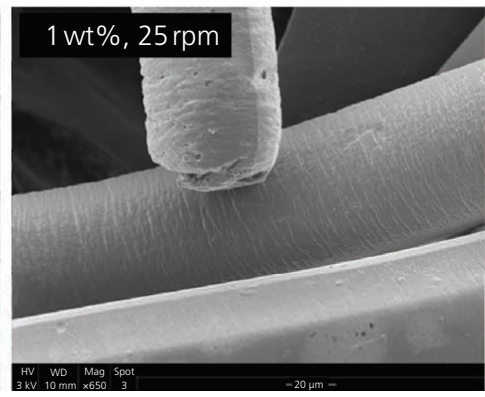

(e)

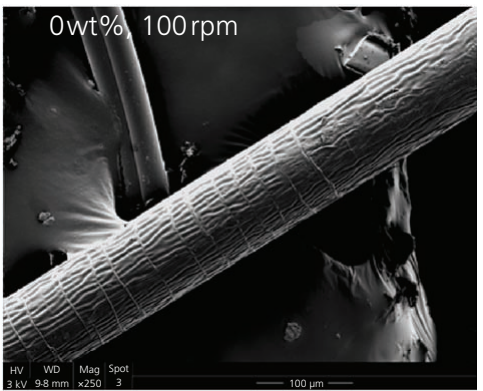

(c)

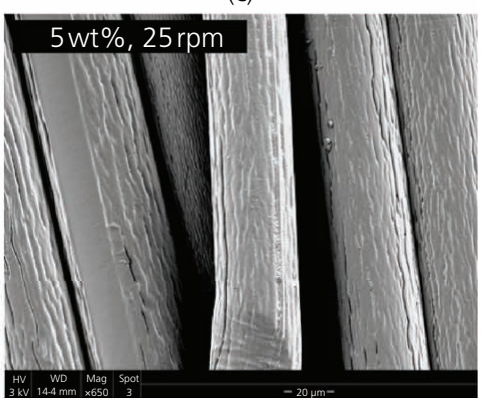

(f)

Figure 2. SEM of fibers to determine surface morphology with (top row) increasing screw speed for 0 wt $\%$ CNC dry-spun polymer and (bottom row) surface morphology with increasing CNC content: (a) 0 wt \%, 1 rpm; (b) 0 wt\%, 15 rpm; (c) 0 wt\%, 100 rpm; (d) 0 wt\%, $25 \mathrm{rpm}$; (e) $1 \mathrm{wt} \%$, $25 \mathrm{rpm}$; (f) $5 \mathrm{wt} \%, 25 \mathrm{rpm}$ 
within the region of the die exit as the result of a cohesive failure and wall-polymer adhesion failure, while severe (melt) fracture is believed to be caused by fluid instabilities within the die which then propagate downstream. ${ }^{25,26}$ Sharkskin melt fracture has been connected to many experimental factors, including temperature, composition, die geometry and extrusion and winding speeds. ${ }^{25-28}$

With those factors in mind, the experiment was designed to facilitate fiber diameter and drawing rather than to tune specific surface morphologies. A concentration of $30 \mathrm{wt} \%$ polymer in solution was chosen after preliminary studies showed stable jet formation, and a maximum temperature of $115^{\circ} \mathrm{C}$ was chosen to facilitate solvent evaporation. In the case of melt-spinning, increasing temperature has been shown to relieve melt fracture as the molecular mobility increases with increasing temperature and thus the polymer will relax more rapidly after stretching and shearing within the die and at the exit. ${ }^{27}$ However, in dry-spinning, increasing temperature also increases solvent evaporation and could result in an increase in concentration, which may have the opposite effect on melt fracture. Both were held constant as the effect of $\mathrm{CNC}$ content was the primary interest. Interestingly, $\mathrm{CNC}$ content did not appear to have a significant effect on surface texture despite some data which suggest that by promoting slip, melt fracture can be mitigated. CNCs are shear thinning and may promote slip, but, in this case, did not appear to reduce melt fracture, but could have shifted the onset of severe sharkskin to lower screw speeds/ extrusion speeds. This is possible because of the increase in solution viscosity with increasing $\mathrm{CNC}$ content at sufficiently high shear rates. Additionally, a conical V-tapered orifice with a diameter of $250 \mu \mathrm{m}$ was employed. It has been shown that zero-length or short-length orifices such as the one employed in the present study can contribute to the onset of melt fracture because the length of the die does not allow sufficient relaxation of the polymer and sharp entry angles create flow instabilities as the polymer is compressed and stretched inside the die. Increasing the extrusion rate or the polymer concentration can exacerbate this effect as the polymer relaxation rate depends on how quickly it is deformed and how high the molecular mobility of the solution is.

\subsection{Crystallinity}

Crystallinity was examined as a function of the processing history and composition in the as-spun fibers by using DSC. Representative DSC thermograms are shown in Figures 3(a) and 3(b). All specimens exhibited a single melting peak despite a double melting peak being common in polylactides and in PLA nanocomposites. ${ }^{6,22,29-32}$ A double melting peak is indicative of melt recrystallization in which imperfect crystals from the process melt at a lower temperature, recrystallize to form new crystals during the heating scan and subsequently remelt at a higher temperature. ${ }^{33}$ This second peak, which occurs at temperatures $5-10^{\circ} \mathrm{C}$ lower than the primary melting peak, was absent in all dry-spun fibers, which, in combination with the high melting point of $166 \pm 1 \cdot 7^{\circ} \mathrm{C}$, suggested that stable crystals formed in the dry-spinning process or during cold crystallization upon heating.

Upon heating, the polymer can undergo crystallization above the glass transition temperature if the polymer reaches a temperature at which the molecular mobility is high enough for molecules to rearrange. ${ }^{32}$ PLA typically exhibits this phenomenon after rapid cooling because molecular chains do not have sufficient time to crystallize. $^{23,32}$ Since total crystallization depends on nucleation and growth rate, both should be maximized to achieve the highest crystallinity possible. Cold crystallization peaks are evident in the DSC thermograms, although the degree of cold crystallization varied significantly with $\mathrm{CNC}$ content and screw speed. In the thermograms, a cold crystallization peak was observed for many

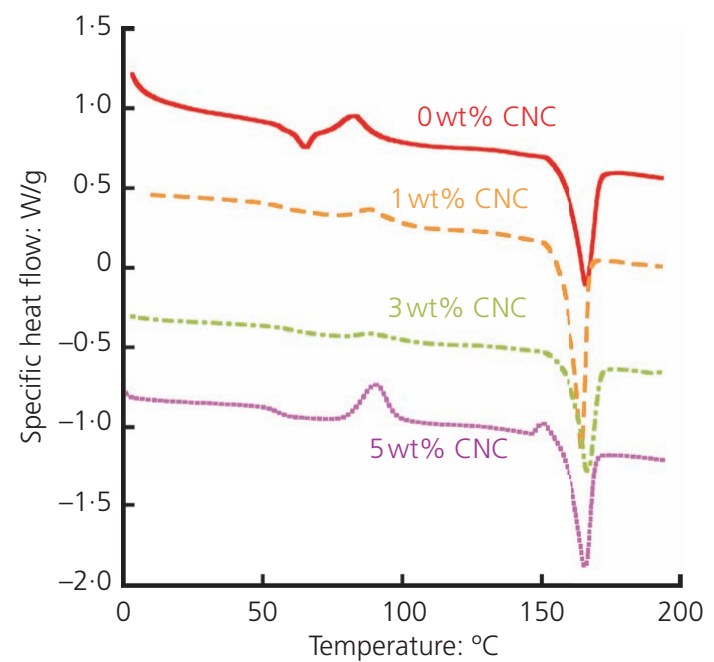

(a)

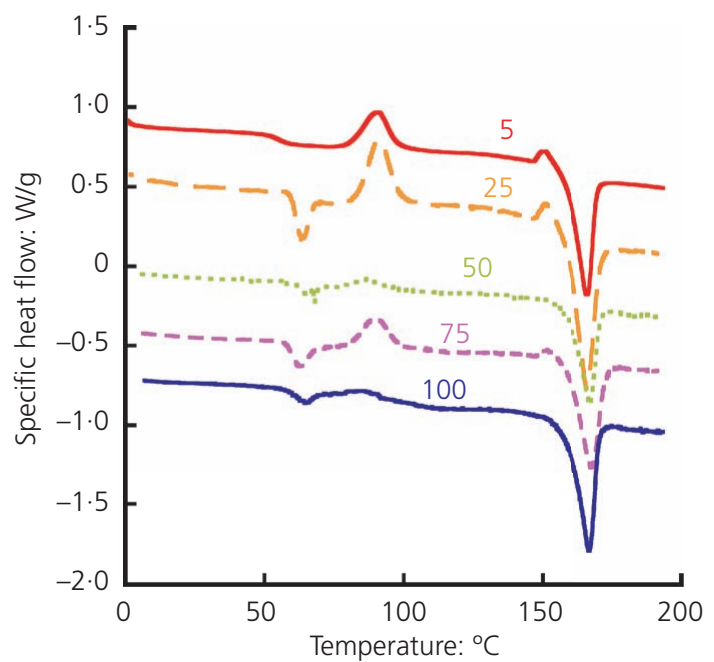

(b)

Figure 3. Representative DSC thermograms of as-spun fiber as a function of (a) CNC content ( $w t \%)$ ( 25 rpm constant) and (b) screw speed (rpm) (5 wt\% CNC constant) 
combinations of conditions, as seen in Figure 3. However, some conditions, such as $5 \mathrm{wt} \% \mathrm{CNC}$ content, produced large cold crystallization peaks, while other conditions produced very small cold crystallization peaks, such as the $1 \mathrm{wt} \% \mathrm{CNC}$ content (Figure 3(a)). Highly crystalline fibers are desirable as the strength and stiffness should both increase with increasing crystallinity. It may be desirable to eliminate or reduce cold crystallization by achieving high crystallinity during fiber spinning. This could reduce time and resources spent on postprocessing steps and avoid shape and property changes that may happen during use if the fiber is heated above its glass transition temperature.

The degree of crystallinity $\chi$ was calculated from Equation 1, where $\Delta H_{\mathrm{m}}$ is the enthalpy of melting, $\Delta H_{\mathrm{c}}$ is the enthalpy of cold crystallization (opposite sign of $\Delta H_{\mathrm{m}}$ ), $\Delta H_{\mathrm{m}}^{\circ}$ is the theoretical maximum enthalpy of melting (assumed to be $93 \mathrm{~J} / \mathrm{g}$ ) and $x_{\mathrm{cnc}}$ is the weight fraction of CNCs in the PLA/CNC fiber. ${ }^{22}$ The cold crystallization temperature $T_{\mathrm{cc}}$ was also taken as the temperature of the maximum specific heat capacity. These data are tabulated in Table 1 in the online supplementary material.

1. $\chi=\frac{\Delta H_{\mathrm{m}}+\Delta H_{\mathrm{c}}}{\Delta H_{\mathrm{m}}^{\circ}\left(1-x_{\mathrm{cnc}}\right)} \times 100 \%$

Figure 4(a) summarizes the effects of CNC content and extrusion speed on as-spun PLA fibers. CNC has been shown to act as a nucleating agent for PLA, which has a very slow rate of crystallization. ${ }^{29,31,33}$ Of the fibers tested, the $1 \mathrm{wt} \% \mathrm{CN}$ content exhibited the highest $\chi$. However, further addition of $\mathrm{CNC}$ reduced $\chi$ as CNCs can inhibit crystallization by acting as a barrier to polymer reorganization; CNCs may act as a physical barrier or inhibit reorganization when weak secondary interactions between the ester group oxygen of PLA and the hydroxyl groups of the CNCs occur. ${ }^{34}$ Weak hydrogen bonding has been thought to inhibit other molecular processes in polymers. ${ }^{34,35}$ This observation appeared true for all screw speeds examined, and the data reached approximately similar degrees of crystallinity. Crystallinity in PLA is facilitated by strain-induced alignment in processes such as fiber spinning and film orientation; so by increasing the amount of spin drawing on the fiber, it was expected that $\chi$ would change. ${ }^{29}$ However, this did not occur. It is possible that for the screw speeds examined, the difference in speed did not produce alignment differences large enough to overcome the slow crystallization rate of PLA, and, therefore, samples achieved approximately the same amount of crystallinity. The variability in fiber thermograms may also be attributed in part to the complex mechanism of solidification during dry-spinning in which evaporation and heat transfer occur simultaneously. Fibers were not drawn and wound in a controlled-atmosphere chamber, but rather the winding process took place under a ventilated hood in an open laboratory, which may have produced variations in the evaporation rate. Regardless of the lack of rate dependence, dry-spinning did produce a significant increase in $\chi$ compared to the cast PLA solution. This can be attributed to the preferential alignment of molecular chains and direct winding of the fibers from the orifice, facilitating drawing of the fibers during processing. Both enhance crystallinity.

$T_{\mathrm{cc}}$ decreased for the dry-spun fibers, as seen in Figure 4(b). $T_{\mathrm{cc}}$ reduced from $133^{\circ} \mathrm{C}$ for the $0 \mathrm{wt} \%$ solution to $84-97^{\circ} \mathrm{C}$ for the

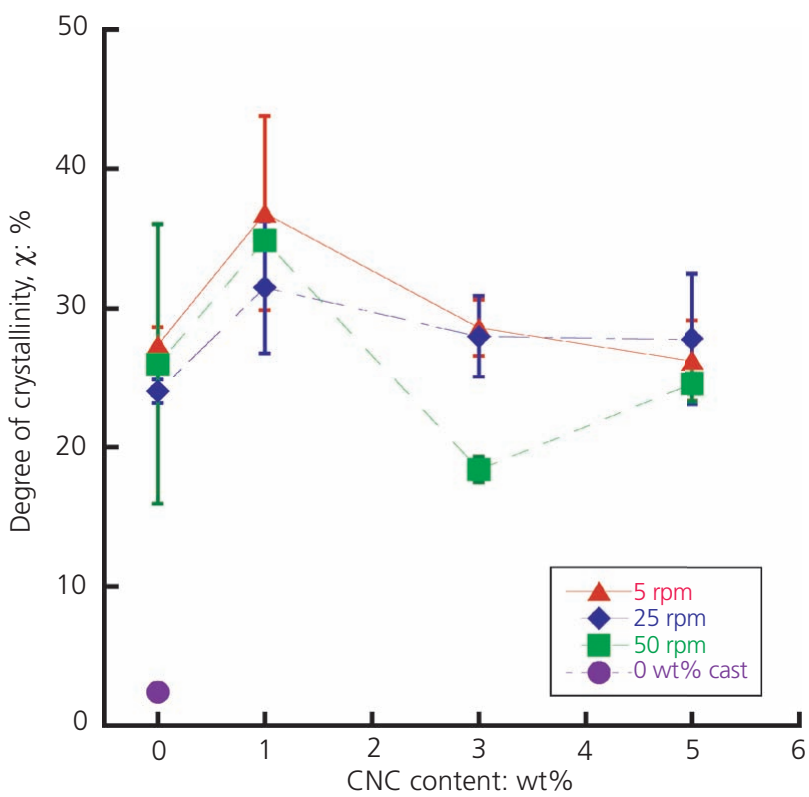

(a)

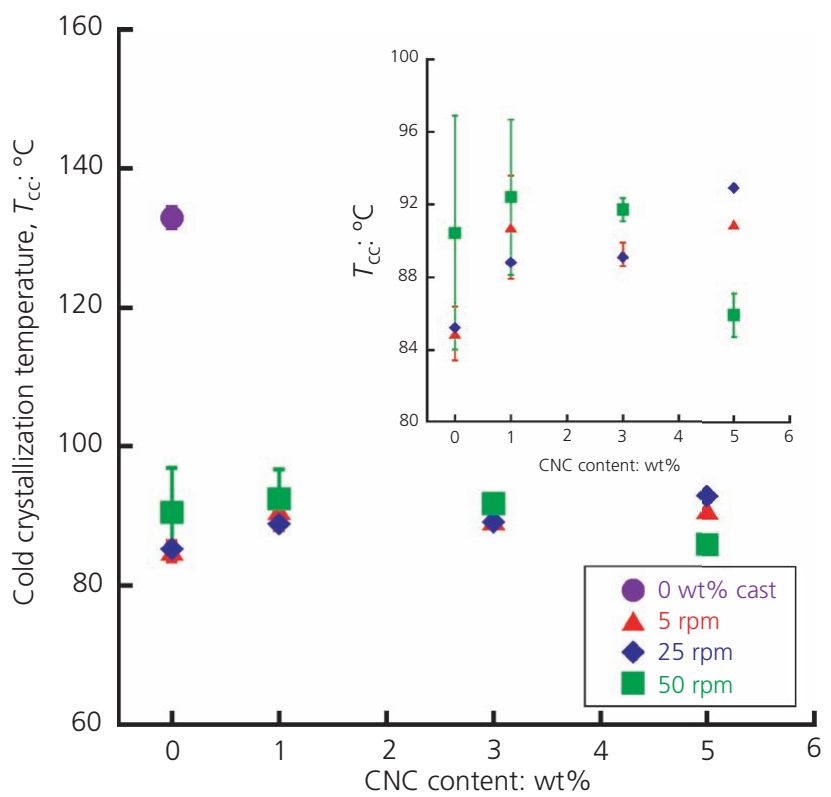

(b)

Figure 4. (a) Degree of crystallinity for as-spun processing history and (b) cold crystallization temperature $T_{\mathrm{cc}}$ with varying CNC wt\% for the first heat (processing history) 
as-spun fibers, with a minimum value of $84^{\circ} \mathrm{C}$ for the $0 \mathrm{wt} \%$ fibers. As seen for $\chi$, where $\mathrm{CNC}$ content inhibited crystallization at $\mathrm{CNC}$ concentrations greater than $1 \mathrm{wt} \%, T_{\mathrm{cc}}$ also began to increase as $\mathrm{CNC}$ content increased for the lowest screw speeds (Figure 4(b) insert). This suggests that even at $1 \mathrm{wt} \%$, the CNCs behaved as a barrier to crystal growth because the temperature at which molecules had sufficient mobility to begin rearranging was suppressed to higher temperatures despite a more conducive initial state from the spinning processing. For small screw speeds, fiber draw would be very large since the polymer is being extruded slowly, but wound onto the barrel quickly. This should have produced more alignment of molecular chains and possibly the $\mathrm{CNC}$ along the tensile axis of the fiber, although the crystallization process during fiber spinning would be competing with higher rates of cooling and solvent evaporation. For crystallinity and $T_{\mathrm{cc}}$, this would imply a higher total crystallinity and a lower $T_{\mathrm{cc}}$ due to the preferential alignment. Of the as-spun fibers, the $50 \mathrm{rpm}$ screw speed showed the most variability in $T_{\mathrm{cc}}$ with $\mathrm{CNC}$ content and the 5 and $25 \mathrm{rpm}$ screw speeds showed only small differences in $T_{\mathrm{cc}}$. The weak dependence on screw speed may be, again, a symptom of variable atmosphere, or it may be that the efficient self-nucleation from solution was sufficient in all cases for fibers to achieve the same level of crystallinity; thus, the dependence on screw speed was weak.

\subsection{Mechanical performance}

A representative stress-strain diagram for each $\mathrm{CNC}$ content is shown in Figure 5(a). The fibers exhibited a mixture of ductile and brittle behavior. Only the $5 \mathrm{rpm}$ condition was used for tensile testing because (a) all solutions produced continuous fibers at this condition and $(b)$ the fibers were less brittle than those produced at faster extrusion speeds and could be handled throughout sample preparation. The fibers were grouped into subsets of 20-35, 40-55 and $60-75 \pm 2 \cdot 5 \mu \mathrm{m}$ diameter.

Increased stiffness and strength were primary motivations for reinforcing PLA with CNC. Factors which affected mechanical performance were crystallinity and $\mathrm{CNC}$ loading. Elastic moduli from 2 to $6.5 \mathrm{GPa}$ were achieved for dry-spun fibers, which is comparable to dry-spun PLA values seen elsewhere (Figure 5(b), Table 2 in the online supplementary material). ${ }^{23}$ Of the three fiber diameter sets, the $40-55 \mu \mathrm{m}$ set shows the clearest trend with $\mathrm{CNC}$ content; it increased linearly with increasing $\mathrm{CNC}$ content and approximately doubles from 0 to $5 \mathrm{wt} \%$. The increase in elastic modulus is primarily due to increasing $\mathrm{CNC}$ content as fiber crystallinity decreased with increasing CNC content, as seen in Figure 4(a). Fibers failed in either a brittle or a ductile manner, without a strong dependence on $\mathrm{CNC}$ content; thus, the strength at failure data are scattered (see Figure 5(c), Table 2 in the online supplementary material). Addition of $\mathrm{CNCs}$ was expected to introduce some brittleness into fibers, particularly at high concentrations, since other studies have observed embrittlement due to the addition of $\mathrm{CNCs}{ }^{8,36}$ For fibers exhibiting ductile failure, the strength at failure is similar to the yield point. These values are comparable to the low end of tensile strength values seen in PLA fibers dry-spun from chloroform. ${ }^{20}$ Interestingly, the average strength

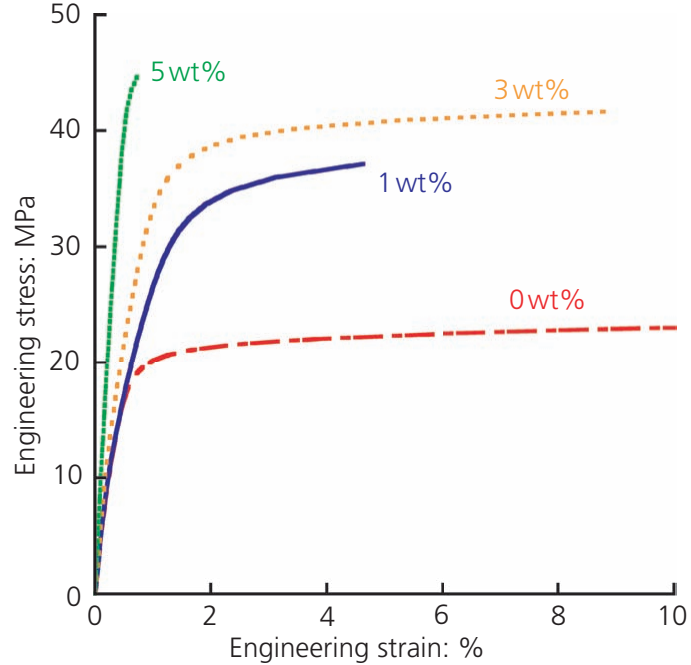

(a)

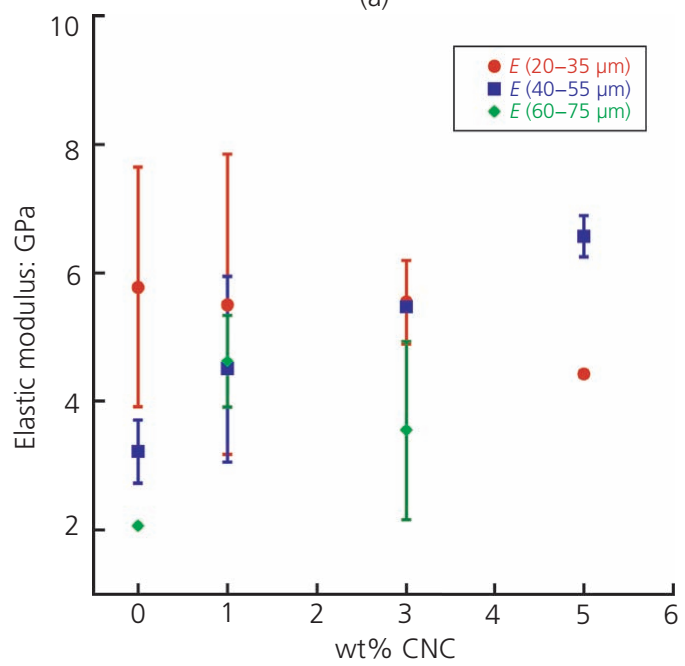

(b)

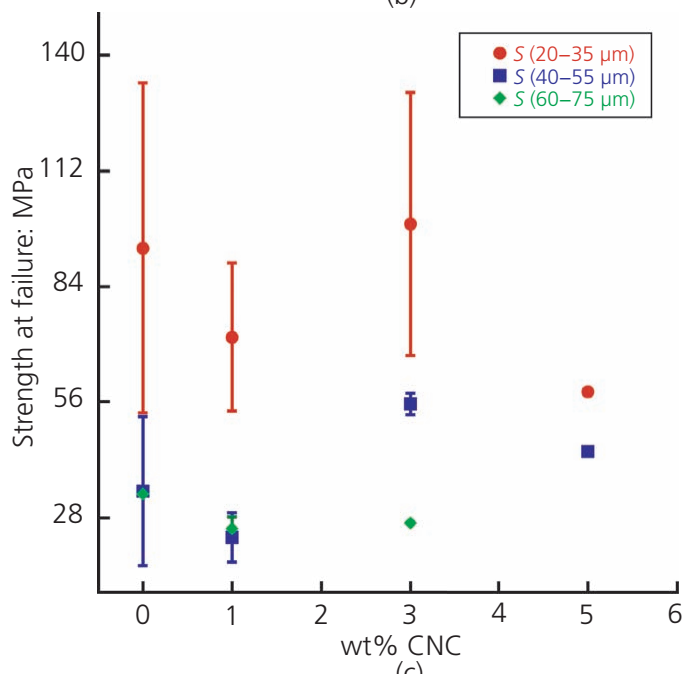

(c)

Figure 5. Mechanical performance of PLAVCNC fiber prepared at 5 rpm screw speed: (a) example tensile stress-strain data; (b) elastic modulus (E) against CNC concentration; (c) ultimate tensile strength against CNC concentration 
at failure for a fiber diameter set decreased with increasing fiber diameter size - that is, the smallest diameters exhibited the highest strength at failure. This agrees with Griffith's failure criterion, which states that the likelihood of a defect being present increases with increasing size, which, in this case, is fiber diameter. ${ }^{37}$

Fibers may have failed due to defects that arise particularly from the dry-spinning process. Defects included porosity and processing defects which resulted in variation in fiber diameter and thus a higher stress at a failure site, as well as surface defects, such as those in Figure 2, that may have initiated premature failure. Dryspinning of PLA with CNC was challenging as more hydrophobic solvents such as chloroform could not be easily employed; thus, a higher temperature solvent was employed. Moreover, residual solvent that was removed during postprocessing could leave behind larger porosity. During processing, fibers were at higher temperature and subjected to an external drawing force that would help polymers diffuse into vacancies as the solvent was removed and close porosity. Postspinning thermal treatment processes were carried out far below the processing temperature and under static conditions.

Continuous and short-fiber micromechanical models were fit to the elastic modulus data for the $40-55 \mu \mathrm{m}$ data to predict the composite elastic modulus $E$. Isostrain and isostress models were fit to the data, as seen in Figure 6. These models assume a parallel or series relationship of the elastic moduli of the fiber and matrix material, respectively. ${ }^{7}$ The Halpin-Tsai model, Equation 2, was also fit to data as previous studies have demonstrated good agreement with data. $^{7,12}$ The geometry factor, in this case, is the aspect ratio $L / D$, $V_{\mathrm{f}}$ is the volume fraction of the fiber and $\eta$ is the ratio defined in the following. The subscripts ' $\mathrm{f}$ ' and ' $\mathrm{m}$ ' stand for fiber and matrix.

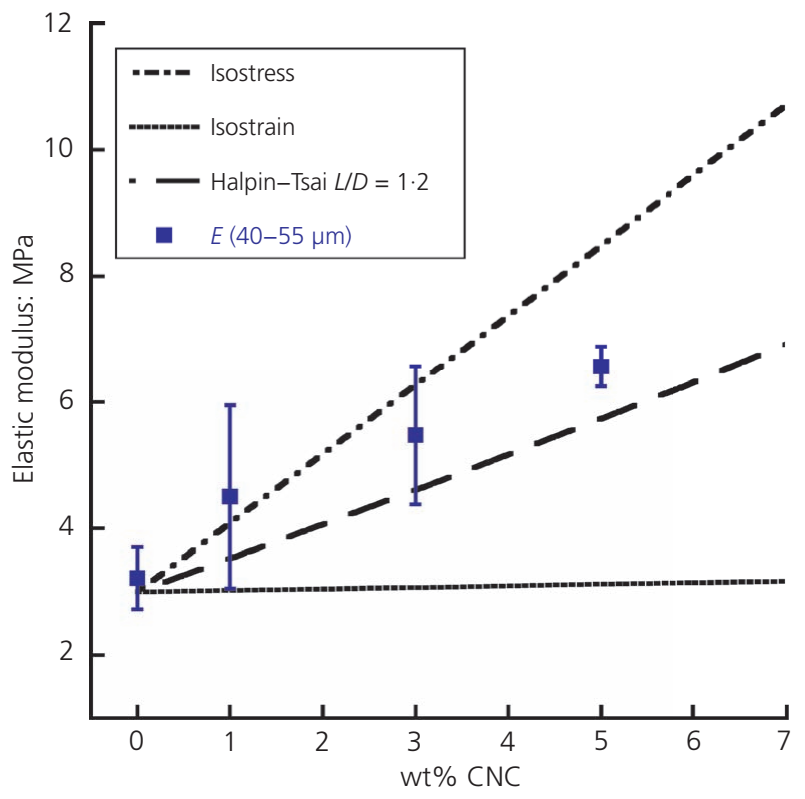

Figure 6. Micromechanical models fit to experimental data
The average aspect ratio was calculated from transmission electron microscope images of the $\mathrm{CNC}$ to find $L / D=11 \cdot 2$.

2. $E=E_{\mathrm{m}}\left[\frac{1+(2 L / D) \eta V_{\mathrm{f}}}{1-\eta V_{\mathrm{f}}}\right]$

where

3. $\eta=\frac{\left(E_{\mathrm{f}} / E_{\mathrm{m}}\right)-1}{\left(E_{\mathrm{f}} / E_{\mathrm{m}}\right)+1}$

The data are between the isostrain and Halpin-Tsai predictions. Error in the Halpin-Tsai predictions may result from too wide a distribution of $L / D$ or aggregation of CNCs changing the effective $L / D .^{7}$ In a previous study, the Halpin-Tsai prediction was empirically fit to obtain a lower $L / D$ ratio, which, in turn, was attributed to the agglomeration of the CNCs in the polymer matrix as agglomeration would result in a lower effective $L / D$. Aggregation was probable as chemical incompatibility between the $\mathrm{CNC}$ and the hydrophobic PLA may inhibit dispersion. ${ }^{7,38}$ However, this is most likely not the case with the present data set as the data lie above the $L / D=11 \cdot 2$ prediction, which implies that $L / D$ is underestimated for the current data set. This suggests a higher effective $L / D$ ratio than what is predicted by the arithmetic average $L / D$ from TEM images. Secondly, the Halpin-Tsai model assumes that short fiber reinforcements are aligned along the primary fiber axis and that there is good adhesion between the matrix and the reinforcement. Poor chemical compatibility between CNCs and PLA may invalidate the assumption of perfect adhesion, although it is difficult to resolve from the data. ${ }^{7}$ Additionally, the Halpin-Tsai model does not accommodate for nanoparticle alignment, which may result in a gross error for anisotropic particles such as CNCs, where the transverse and axial properties can vary by as much as an order of magnitude. ${ }^{1}$

Crystallinity was not incorporated into any of the micromechanical models, which may also explain why the models underpredict the elastic modulus at certain concentrations of $\mathrm{CNC}$, particularly at $1 \mathrm{wt} \%$ CNC. Crystalline and amorphous regions display different densities and elastic properties. As crystallinity increases, the elastic modulus and strength of polymers tend to increase as well. If the crystallinity content was assumed to be constant, which is not the case here, the assumptions of the isostrain, isostress and Halpin-Tsai models would be valid as all three models assume the elastic modulus of the control as $E_{\mathrm{m}}$. However, this assumption is not valid for the present study as $\chi$ increased for $1 \mathrm{wt} \%$ and decreased with increasing $\mathrm{CNC}$ content. However, some contribution from the increased crystallinity may account for the vertical shift up from the Halpin-Tsai predictions as $\mathrm{CNC}$ acts as a nucleation agent. ${ }^{7,12}$

\section{Conclusion}

Dry-spinning was investigated as a potential method for producing CNC/PLA fibers from DMF. Surface morphology 
differences were observed in fibers with and without $\mathrm{CNC}$ as the screw speed increased, assumed here to be proportional to the extrusion speed. The onset of severe melt fracture occurred at high screw speeds, and the addition of CNC did not significantly change the surface texture of fibers produced in the present study. The as-spun crystallinity of fibers was found to not depend heavily on processing conditions, however, small differences in the degree of crystallinity were observed that agree with previous observations on CNC in PLA. Dry-spinning produced fibers with moduli between 2 and $6.5 \mathrm{GPa}$ and a maximum strength at failure of $100 \mathrm{MPa}$. However, there is a significant scatter in the data with a shift from a ductile to a brittle-like behavior as the CNC content increases and as a result of premature failure most likely due to processing defects from the spinning process.

\section{Acknowledgements}

The authors would like to acknowledge financial support from the Private-Public Partnership for Nanotechnology in the Forestry Sector (P3Nano) under Grant Number 107528 and National Science Foundation Integrative Graduate Education and Research Traineeship: Sustainable Electronics Grant (Grant Number 1144843).

\section{REFERENCES}

1. Moon RJ, Martini A, Nairn J, Simonsen J and Youngblood J (2011) Cellulose nanomaterials review: structure, properties and nanocomposites. Chemical Society Reviews 40(7): 3941-3994.

2. Davis CS, Grolman DL, Karim A and Gilman JW (2015) What do we still need to understand to commercialize cellulose nanomaterials? Green Materials 3(3): 53-58, https://doi.org/10.1680/jgrma.15.00013.

3. Trache D, Hussin MH, Haafiz MKM and Thakur VK (2017) Recent progress in cellulose nanocrystals: sources and production. Nanoscale 9(5): 1763-1786.

4. Shrestha S, Diaz JA, Ghanbari S and Youngblood JP (2017) Hygroscopic swelling determination of cellulose nanocrystal (CNC) films by polarized light microscopy digital image correlation. Biomacromolecules 18(5): 1482-1490.

5. Diaz JA, Ye Z, Wu X et al. (2014) Thermal conductivity in nanostructured films: from single cellulose nanocrystals to bulk films. Biomacromolecules 15(11): 4096-4101.

6. Diaz JA, Wu X, Martini A, Youngblood JP and Moon RJ (2013) Thermal expansion of self-organized and shear-oriented cellulose nanocrystal films. Biomacromolecules 14(8): 2900-2908.

7. Peng J, Ellingham T, Sabo R, Turng LS and Clemons CM (2014) Short cellulose nanofibrils as reinforcement in polyvinyl alcohol fiber. Cellulose 21(6): 4287-4298.

8. Chen S, Schueneman G, Pipes RB and Moon RJ (2014) Effects of crystal orientation on cellulose nanocrystals-cellulose acetate nanocomposite fibers prepared by dry spinning. Biomacromolecules 15(10): 3827-3835.

9. Chang H, Luo J, Liu HC et al. (2017) Ductile polyacrylonitrile fibers with high cellulose nanocrystals loading. Polymer 122: 332-339.

10. Chang H, Chien AT, Liu HC et al. (2015) Gel spinning of polyacrylonitrile/cellulose nanocrystal composite fibers. ACS Biomaterials Science \& Engineering 1(7): 610-616.

11. Peresin MS, Habibi Y, Zoppe JO, Pawlak JJ and Rojas OJ (2010) Nanofiber composites of polyvinyl alcohol and cellulose nanocrystals: manufacture and characterisation. Biomacromolecules 11(3): 674-681.

12. Ureña-Benavides EE, Brown PJ, Kitchens CL et al. (2011) Structure and mechanical properties of wet-spun fibers made from natural cellulose nanofibers. Biomacromolecules 122(1): 13022-13028.
13. Ureña-Benavides EE and Kitchens CL (2012) Cellulose nanocrystal reinforced alginate fibers - biomimicry meets polymer processing. Molecular Crystals and Liquid Crystals 556(1): 275-287.

14. Endo R, Saito T and Isogai A (2013) TEMPO-oxidized cellulose nanofibril/poly(vinyl alcohol) composite drawn fibers. Polymer 54(2): 935-941.

15. Uddin AJ, Araki J and Gotoh Y (2011) Toward 'strong' green nanocomposites: polyvinyl alcohol reinforced with extremely oriented cellulose whiskers. Biomacromolecules 12(3): 617-624.

16. Eling B, Gogolewski S and Pennings AJ (1982) Biodegradable materials of poly(L-lactic acid): 1. melt-spun and solution-spun fibres. Polymer 23(11): 1587-1593.

17. Leenslag JW and Pennings AJ (1987) High-strength poly(L-lactide) fibres by a dry-spinning/hot-drawing process. Polymer 28(10): 1695-1702.

18. Horacek I and Kalisek (1994) Polylactide: II. discontinuous dry spinning: hot drawing preparation of fibers. Journal of Applied Polymer Science 54(11): 1759-1765.

19. Postema AR, Luiten AH and Pennings AJ (1990) High-strength poly (L-lactide) fibers by a dry-spinning/hot-drawing process: I. influence of the ambient temperature on the dry-spinning process. Journal of Applied Polymer Science 39(6): 1265-1274.

20. Postema AR and Pennings AJ (1989) Study on the drawing behavior of poly(L-lactide) to obtain high-strength fibers. Journal of Applied Polymer Science 37(8): 2351-2369.

21. Horacek I and Kalisek V (1994) Poylactide: I. continuous dry spinning-hot drawing preparation of fibers. Journal of Applied Polymer Science 54(11): 1751-1757.

22. Gogolewski S and Pennings AJ (1984) Resorbable materials of poly(L-lactide): II. fibers spun from solutions of poly(L-lactide) in good solvents. Journal of Applied Polymer Science 28(3): 1045-1061.

23. Sullivan EM, Moon RJ and Kalaitzidou K (2015) Processing and characterization of cellulose nanocrystals/polylactic acid nanocomposite films. Materials (Basel) 8(12): 8106-8116.

24. Gupta B, Revagade N and Hilborn J (2007) Poly(lactic acid) fiber: an overview. Progress in Polymer Science 32(4): 455-482.

25. Ilankeeran PK, Mohite PM and Kamle S (2012) Axial tensile testing of single fibres. Modern Mechanical Engineering 2(4): 151-156.

26. Larson RG (1992) Instabilities in viscoelastic flows. Rheologica Acta 31(3): 213-263.

27. Migler KB, Son Y, Qiao F and Flynn K (2002) Extensional deformation, cohesive failure, and boundary conditions during sharkskin melt fracture. Journal of Rheology 46(2): 383-400.

28. Miller E and Rothstein JP (2004) Control of the sharkskin instability in the extrusion of polymer melts using induced temperature gradients. Rheologica Acta 44(2): 160-173.

29. El Kissi N, Piau JM and Toussaint F (1997) Sharkskin and cracking of polymer melt extrudates. Journal of Non-Newtonian Fluid Mechanics 68(2-3): 271-290.

30. Li H and Huneault MA (2007) Effect of nucleation and plasticization on the crystallization of poly(lactic acid). Polymer 48(23): 6855-6866.

31. Martin OA (2001) Poly(lactic acid): plasticization and properties of biodegradable multiphase systems polymer. Polymer 42(14): 6209-6219.

32. Habibi Y, Aouadi S, Raquez JM and Dubois P (2013) Effects of interfacial stereocomplexation in cellulose nanocrystal-filled polylactide nanocomposites. Cellulose 20(6): 2877-2885.

33. Sarasua JR, Prud'homme RE, Wisniewski M, Le Borgne A and Spassky N (1998) Crystallization and melting behavior of polylactides. Macromolecules 31(12): 3895-3905.

34. Yasuniwa M, Tsubakihara S, Sugimoto Y and Nakafuku C (2004) Thermal analysis of the double-melting behavior of poly(L-lactic acid). Journal of Polymer Science Part B: Polymer Physics 42(1): 25-32.

35. Peng SX, Moon RJ and Youngblood JP (2014) Design and characterization of cellulose nanocrystal-enhanced epoxy hardeners. Green Materials 2(4): 193-205, https://doi.org/10.1680/gmat.14.00015. 
Green Materials

Volume 6 Issue GMAT1
Dry-spinning of cellulose nanocrystal/

polylactic acid composite fibers

Clarkson and Youngblood
36. Urena-Benavides EE, Brown PJ and Kitchens CL (2010) Effect of jet stretch and particle load on cellulose nanocrystal-alginate nanocomposite fibers. Langmuir 26(17): 14263-14270.

37. Liu JC, Martin DJ, Moon RJ and Youngblood JP (2015) Enhanced thermal stability of biomedical thermoplastic polyurethane with the addition of cellulose nanocrystals. Journal of Applied Polymer Science 132(22): 1-8.

38. Oksman K, Aitomäki Y, Mathew AP et al. (2016) Review of the recent developments in cellulose nanocomposite processing.

Composites Part A: Applied Science and Manufacturing 83(1): 2-18.

\section{How can you contribute?}

To discuss this paper, please submit up to 500 words to the journal office at journals@ice.org.uk. Your contribution will be forwarded to the author(s) for a reply and, if considered appropriate by the editor-in-chief, it will be published as a discussion in a future issue of the journal.

ICE Science journals rely entirely on contributions from the field of materials science and engineering. Information about how to submit your paper online is available at www.icevirtuallibrary.com/page/authors, where you will also find detailed author guidelines. 